This document is the accepted manuscript version of the following article:

Seibold, S., Gossner, M. M., Simons, N. K., Blüthgen, N., Müller, J., Ambar11, D., ... Weisser, W. W. (2019). Arthropod decline in grasslands and forests is associated with 1andscape-1eve1 drivers. Nature, 574(7780), 671-674.

https://doi .org/10.1038/s41586-019-1684-3

\title{
Arthropod decline in grasslands and forests is associated with
}

\section{drivers at landscape level}

Sebastian Seibold ${ }^{1,2 *}$, Martin M. Gossner ${ }^{3}$, Nadja K. Simons ${ }^{1,4}$, Nico Blüthgen ${ }^{4}$, Jörg Müller $^{2,5}$, Didem Ambarlı ${ }^{1,6}$, Christian Ammer ${ }^{7}$, Jürgen Bauhus ${ }^{8}$, Markus Fischer ${ }^{9}$, Jan C. Habel ${ }^{1,10}$, Karl Eduard Linsenmair ${ }^{11}$, Thomas Nauss ${ }^{12}$, Caterina Penone ${ }^{9}$, Daniel Prati $^{9}$, Peter Schall ${ }^{7}$, Ernst-Detlef Schulze ${ }^{13}$, Juliane Vogt $^{1}$, Stephan Wöllauer ${ }^{12}$, Wolfgang W. Weisser ${ }^{1}$

Affiliations:

${ }^{1}$ Terrestrial Ecology Research Group, Department of Ecology and Ecosystem Management, Technical University of Munich, Hans-Carl-von-Carlowitz-Platz 2, 85354 Freising, Germany

${ }^{2}$ Field Station Fabrikschleichach, Department of Animal Ecology and Tropical Biology, Julius-Maximilians-University Würzburg, Glashüttenstraße 5, 96181 Rauhenebrach, Germany

${ }^{3}$ Forest Entomology, Swiss Federal Research Institute WSL, Zürcherstrasse 111, 8903 Birmensdorf, Switzerland

${ }^{4}$ Ecological Networks, Department of Biology, Technical University of Darmstadt, Schnittspahnstraße 3, 64287 Darmstadt, Germany

${ }^{5}$ Bavarian Forest National Park, Freyunger Str. 2, 94481 Grafenau, Germany

${ }^{6}$ Department of Agricultural Biotechnology, Faculty of Agricultural and Natural Sciences, Düzce University, 81620 Düzce, Turkey 
${ }^{7}$ Silviculture and Forest Ecology of the Temperate Zones, University of Göttingen, Büsgenweg 1, 37077 Göttingen, Germany

${ }^{8}$ Chair of Silviculture, Institute of Forest Sciences, Faculty of Environment and Natural Resources, University of Freiburg, Tennenbacherstr. 4, 79106 Freiburg, Germany

${ }^{9}$ Institute of Plant Sciences, University of Bern, Altenbergrain 21, 3013 Bern, Switzerland

${ }^{10}$ Evolutionary Zoology, Biosciences, Salzburg University, Hellbrunner Str. 34, 5020 Salzburg, Austria

${ }^{11}$ Department of Animal Ecology and Tropical Biology, Julius-Maximilians-University Würzburg, Am Hubland, 97074 Würzburg, Germany

12 Faculty of Geography, Philipps-Universität Marburg, Deutschhausstr. 10, 35032 Marburg, Germany

${ }^{13}$ Max Planck Institute for Biogeochemistry, Box 100164, 07701 Jena, Germany

${ }^{*}$ Corresponding author: sebastian.seibold@tum.de 


\section{Summary}

Recent reports on local extinctions of arthropod species $^{1}$ and of massive declines in arthropod biomass ${ }^{2}$ point to land-use intensification as a major driver of decreasing biodiversity. However, there are no multi-site time-series of arthropod occurrences across land-use intensity gradients to confirm causal relationships. Moreover, it remains unclear which land-use types and arthropod groups are affected and whether the observed declines in biomass and diversity are linked to one another and continue.

Here we analyzed arthropod data on more than 1 million individuals and 2,700 species from standardized inventories from 2008 to 2017 at 150 grassland and 140 forest sites in three regions of Germany. Overall gamma diversity in grasslands and forests decreased over time indicating loss of species across sites and regions. In annually sampled grasslands, biomass, abundance and species number of arthropods declined by $67 \%, 78 \%$, and $34 \%$, respectively. The decline was consistent across trophic levels, mainly affected rare species, and its magnitude was independent of local land-use intensity. However, sites embedded in landscapes with higher cover of agricultural land showed a stronger temporal decline. In $\mathbf{3 0}$ forest sites with annual inventories, biomass and species number, but not abundance, decreased by $41 \%$ and $36 \%$, respectively. This was supported by analyses of all forest sites sampled in 3year intervals. The decline affected rare and abundant species and trends differed across trophic levels.

Our results show that there are widespread declines in arthropods that concern biomass, abundance and diversity across trophic levels. Declines in forests demonstrate that arthropod loss is not restricted to open habitats. Our results 
suggest that major drivers of arthropod decline act at larger spatial scales, and are, at least for grasslands, associated with agriculture at the landscape level. This implies that land-use relevant policies need to address the landscape scale to mitigate negative effects of land-use practices.

\section{Main text}

Much of the debate on the human-induced biodiversity crisis has focused on vertebrates $^{3}$, yet population decline and extinctions may be even more substantial in small organisms such as terrestrial arthropods ${ }^{4}$. Recent studies report declines in biomass of flying insects ${ }^{2}$, diversity of insect pollinators ${ }^{5,6}$, butterflies and moths $s^{1,7-10}$, hemipterans ${ }^{11,12}$ and beetles ${ }^{7,13,14}$. Owing to the associated negative effects on food webs $^{15}$, ecosystem functioning and ecosystem services ${ }^{16}$, the insect loss has spurred an intense public debate. However, time-series data on arthropods are rather limited and studies have so far focused on a small range of taxa ${ }^{11,13,14}$, few land-use and habitat types ${ }^{12}$ or even on single sites ${ }^{1,17}$. In addition, many studies lack species information $^{2}$ or high temporal resolution ${ }^{2,12}$. Hence, it remains unclear whether reported declines in arthropods are a general phenomenon that is driven by similar mechanisms across land-use types, taxa and functional groups.

The reported declines are suspected to be mainly caused by human land use ${ }^{2}$. Locally, farming practices can affect arthropods directly by application of insecticides ${ }^{18,19}$, mowing ${ }^{20}$ or soil disturbance, or indirectly via changes in plant communities through application of herbicides or fertilizer ${ }^{21}$. Similarly, forestry practices can affect local arthropod communities via changes in tree species composition or forest structure ${ }^{22}$. In addition, local arthropod populations can be affected by land use in the surrounding landscape, e.g. through drift and transport of pesticides and nitrogen by air or 
water ${ }^{23,24}$, through effects of habitat loss on meta-communities (i.e. source-sink dynamics ${ }^{25}$ ), or by hampering dispersal.

To disentangle local and landscape-level effects of land use on temporal trends in arthropod communities of grasslands and forests, we used data from the Biodiversity Exploratories research program, including more than 1 million individuals and 2,700 arthropod species (Extended Data Table S1). Arthropods were collected annually at 150 grassland sites by standardized sweep-net sampling in June and August from 2008 to 2017 and at 30 forest sites with flight-interception traps over the whole growing period from 2008 to 2016. An additional 110 forest sites were sampled in 2008, 2011 and 2014 to test for trends across a larger number of sites. Both grassland and forest sites cover gradients in local land-use intensity. Land-use intensity was quantified in the form of compound indices based on grazing, mowing and fertilization intensity in grasslands ${ }^{26}$, and on recent biomass removal, the proportion of non-natural tree species and deadwood origin in forests ${ }^{27}$. To analyze landscape-level effects, we quantified the cover of arable fields, grassland and forest in circles with a radius of 250 $\mathrm{m}-2 \mathrm{~km}$ around each sampling site. We modelled temporal trends in arthropod biomass (estimated from body size, see methods), abundance and species number separately for grasslands and forests, and tested for effects of local and landscapescale land-use intensity on these trends, accounting for weather conditions. Analyses were conducted for all species together or separately for different dispersal and trophic guilds.

The total number of arthropod species across all sites, i.e. gamma diversity, was substantially lower in later than in earlier years in both forests and grasslands (Figure 1). Arthropod numbers per site fluctuated over time but revealed an overall decrease with strongest declines from 2008 to 2010 (Figure 1). Year-to-year fluctuations in 
arthropod numbers were partially explained by weather conditions (Extended Data Figure S1, Supplementary Table S1-1 and Supplementary Information Section 2). Accounting for weather, fitted trends from our models showed declines in biomass of $67 \%$ for grasslands and $41 \%$ for forests, declines in species numbers of $34 \%$ for grasslands and $36 \%$ for forests, and declines in abundance of $78 \%$ for grasslands, with no significant change in abundances for forests (-17\%; Figure 1; Supplementary Table S3-1). In grasslands, declines occurred consistently across all trophic guilds (i.e. herbivores, myceto-/detritivores, omnivores and carnivores) although the trend for carnivores was not significant (Supplementary Table S1-1). In forests, the patterns were more complex, with herbivores showing an increase in abundance and species number, while all other trophic guilds declined. Temporal trends of arthropods based on data recorded at 3-year intervals from all 140 forest sites were similar to those based on 30 sites with annual data (Supplementary Table S1-1). Sensitivity analyses removing or reshuffling years showed that the decline was influenced, but not solely dependent on high arthropod numbers in 2008. Fluctuations in numbers including 2008 appear to match trends observed in other studies ${ }^{2}$ suggesting that the recent decline is part of a longer-term trend since at least the early 1990s (Extended Data Figure S2; Supplementary Information Section 3). Further sensitivity analyses showed consistent declines when data of individual sampling dates were not aggregated per year and that declines concerned all three regions (Supplementary Tables S3-2, S3-3 and Figure S3-1).

Linking changes in biomass, abundance and species numbers to one another allows further inferences on mechanisms driving arthropod declines. In grasslands, both abundant and less abundant species declined in abundance (Figure 2), but species loss occurred mostly among less frequent species (Figure 1; Extended Data Figure 
S3; Supplementary Information Section 4). This suggests that the decline in species numbers in grasslands was mainly attributable to a loss of individuals among rare species. In forests, initially less abundant species decreased in abundance, while some of the most abundant species, including invasive species and potential pest species, increased in abundance (Figure 2; Supplementary Table S5-1). The loss of species was, however, irrespective of their frequency (Figure 1; Extended Data Figure S3; Supplementary Information Section 4). This suggests that arthropod decline in forests is driven by mechanisms which negatively affect abundances of many species leading to an overall decline in biomass and species number, but favors some other species which are able to compensate declines in abundances.

The magnitudes of declines in biomass, abundance and species numbers of arthropod communities were independent of local land-use intensity (Supplementary Table S11) as well as changes in plant communities (Supplementary Information Section 6) at all sites. In forests, however, declines in species numbers were weaker at sites with high natural or anthropogenic tree mortality, possibly due to increased local habitat heterogeneity (Extended Data Figure S4). Landscape composition had no effect on arthropod trends in forests (but see limited range in landscape variables; Extended Data Figure S5), but mediated declines in species number in grasslands: the magnitude of the declines increased with increasing cover of arable fields and marginally with cover of grasslands in the surrounding landscape (Figure 3; Supplementary Table S1-1). This suggests that major drivers of arthropod decline in grasslands are associated with agricultural land-use at the landscape scale.

The interaction between a species and the landscape around its habitat depends on its dispersal ability, ultimately determining its occurrence and persistence ${ }^{28}$. In grasslands, taxa of high and low dispersal ability (see methods) declined similarly but 
increasing cover of arable fields (but not of grasslands) in the surroundings amplified biomass declines of weak dispersers more strongly than of strong dispersers (Figure 3; Supplementary Table S7-1). Weak dispersers may experience higher mortality during dispersal and thus have a lower chance of (re)colonization of a particular site when arable field cover is high. In forests, only strong dispersers declined in biomass, abundance and species number over time, whereas weak dispersers increased in abundance and biomass, but less strongly when grassland cover in the landscape was high (Supplementary Table S7-1). This suggests that drivers behind arthropod declines in forests also act at larger spatial scales.

We showed that arthropods declined dramatically not only in biomass, but also in abundance and species numbers, affecting taxa of most trophic levels in both grasslands and forests. Declines in gamma diversity suggest that species might disappear across regions. Our results also indicate that major drivers of arthropod decline in both habitat types act at larger spatial scales, but declines may be moderated by increases in local habitat heterogeneity in forests. While drivers of arthropod decline in forests remain unclear, in grasslands they are associated with the proportion of agricultural land at the landscape-level. However, we cannot ascertain whether the observed declines are driven by recent agricultural intensification at the landscapelevel, e.g. decrease of fallow land and plant-species rich field margins, increased use of pesticides or use of more potent insecticides, or by legacy effects of historic landuse intensification (Supplementary Information Section 3). Time-series data on changes in agrochemical usage or presence of fine-scale arthropod habitats would be necessary to answer this question. Furthermore, it is unclear to what extent changes in climate have reinforced the observed arthropod trends (Supplementary Information Section 2). Our results show that widespread arthropod declines have occurred in 
recent years. Although declines were less pronounced during the second half of our study period, there is no indication that negative trends have been reversed by different measures that have been implemented in recent years. This calls for a paradigm shift in land-use policy at national and international levels to counteract species decline in open and forested habitats with measures that are coordinated across landscapes and regions. Such strategies should aim at improving habitat quality for arthropods and mitigate negative effects of land-use practices across large and continuous areas and not only locally within isolated patches embedded in an inhospitable agricultural matrix. 


\section{References}

1. Habel, J. C. et al. Butterfly community shifts over two centuries. Conserv. Biol. 30, 754-762 (2016).

2. Hallmann, C. A. et al. More than 75 percent decline over 27 years in total flying insect biomass in protected areas. PLoS One 12, e0185809 (2017).

3. Ceballos, G. et al. Accelerated modern human - induced species losses:

Entering the sixth mass extinction. Sci. Adv. 1, 1-6 (2015).

4. Thomas, J. A. et al. Comparative losses of British butterflies, birds, and plants and the global extinction crisis. Science. 303, 1879-1881 (2004).

5. Biesmeijer, J. C. et al. Parallel declines in pollinators and insect-pollinated plants in Britain and the Netherlands. Science. 313, 351-355 (2006).

6. Potts, S. G. et al. Global pollinator declines: Trends, impacts and drivers. Trends Ecol. Evol. 25, 345-353 (2010).

7. Dirzo, R. et al. Defaunation in the Anthropocene. Science. 401, 401-406 (2014).

8. Conrad, K. F., Warren, M. S., Fox, R., Parsons, M. S. \& Woiwod, I. P. Rapid declines of common, widespread British moths provide evidence of an insect biodiversity crisis. Biol. Conserv. 132, 279-291 (2006).

9. Maes, D. \& Van Dyck, H. Butterfly diversity loss in Flanders (north Belgium): Europe's worst case scenario? Biol. Conserv. 99, 263-276 (2001).

10. Stefanescu, C., Torre, I., Jubany, J. \& Páramo, F. Recent trends in butterfly populations from north-east Spain and Andorra in the light of habitat and 
climate change. J. Insect Conserv. 15, 83-93 (2011).

11. Schuch, S., Wesche, K. \& Schaefer, M. Long-term decline in the abundance of leafhoppers and planthoppers (Auchenorrhyncha) in Central European protected dry grasslands. Biol. Conserv. 149, 75-83 (2012).

12. Schuch, S., Bock, J., Krause, B., Wesche, K. \& Schaefer, M. Long-term population trends in three grassland insect groups: A comparative analysis of 1951 and 2009. J. Appl. Entomol. 136, 321-331 (2012).

13. Brooks, D. R. et al. Large carabid beetle declines in a United Kingdom monitoring network increases evidence for a widespread loss in insect biodiversity. J. Appl. Ecol. 49, 1009-1019 (2012).

14. Desender, K., Dekoninck, W., Dufrêne, M. \& Maes, D. Changes in the distribution of carabid beetles in Belgium revisited: Have we halted the diversity loss? Biol. Conserv. 143, 1549-1557 (2010).

15. Bowler, D. E., Heldbjerg, H., Fox, A. D., de Jong, M. \& Böhning-Gaese, K. Long-term declines of European insectivorous bird populations and potential causes. Conserv. Biol. (2019).

16. Siemann, E. \& Weisser, W. W. (eds.) Insects and Ecosystem Function. (Springer, 2004).

17. Shortall, R. C. et al. Long-term changes in the abundance of flying insects. Insect Conserv. Divers. 2, 251-260 (2009).

18. Geiger, F. et al. Persistent negative effects of pesticides on biodiversity and biological control potential on European farmland. Basic Appl. Ecol. 11, 97-105 (2010). 
19. Ewald, J. A. et al. Influences of extreme weather, climate and pesticide use on invertebrates in cereal fields over 42 years. Glob. Chang. Biol. 21, 3931-3950 (2015).

20. Gossner, M. M. et al. Land-use intensification causes multitrophic homogenization of grassland communities. Nature 540, 266-269 (2016).

21. Haddad, N. M., Haarstad, J. \& Tilman, D. The effects of long-term nitrogen loading grassland insect communities. Oecologia 124, 73-84 (2000).

22. Penone, C. et al. Specialisation and diversity of multiple trophic groups are promoted by different forest features. Ecol. Lett. 22, 170-180 (2019).

23. Brittain, C. A., Vighi, M., Bommarco, R., Settele, J. \& Potts, S. G. Impacts of a pesticide on pollinator species richness at different spatial scales. Basic Appl. Ecol. 11, 106-115 (2010).

24. de Jong, F. M. W., de Snoo, G. R. \& van de Zande, J. C. Estimated nationwide effects of pesticide spray drift on terrestrial habitats in the Netherlands. J. Environ. Manage. 86, 721-730 (2008).

25. Thomas, J. A. et al. The quality and isolation of habitat patches both determine where butterflies persist in fragmented landscapes. Proc. R. Soc. B Biol. Sci. 268, 1791-1796 (2001).

26. Blüthgen, N. et al. A quantitative index of land-use intensity in grasslands: Integrating mowing, grazing and fertilization. Basic Appl. Ecol. 13, 207-220 (2012).

27. Kahl, T. \& Bauhus, J. An index of forest management intensity based on assessment of harvested tree volume, tree species composition and dead 
wood origin. Nat. Conserv. 7, 15-27 (2014).

28. Tscharntke, T. et al. Landscape moderation of biodiversity patterns and processes - eight hypotheses. Biol. Rev. 87, 661-685 (2012).

29. Chao, A. et al. Rarefaction and extrapolation with Hill numbers: a framework for sampling and estimation in species diversity studies. Ecol. Monogr. 84, 45-67 (2014).

30. Schenker, N. \& Gentleman, J. F. On judging the significance of differences by examining the overlap between confidence intervals. Am. Stat. 55, 182-186 (2001).

\section{Figure Legends}

Figure 1 | Temporal trends in arthropod communities. a-d, Gamma diversity (i.e. the total number of species across all grassland or forest sites), biomass, abundance and species numbers of arthropods were recorded in 30 forest and 150 grassland sites across Germany. Gamma diversity shows mean incidence-based bias-corrected diversity estimates (Chao's BSS ${ }^{29}$ ) for $\mathrm{q}=0$ and $95 \%$-confidence intervals derived from bootstrapping $(n=200)$. Non-overlapping confidence intervals indicate significant difference ${ }^{30}$. Boxplots show raw data per site and year $\left(n_{\text {grassland }}=1406, n_{\text {forest }}=266\right.$ independent samples). Solid lines indicate significant temporal trends $(p<0.05)$ based on (generalized) linear mixed models that included weather conditions, and local and landscape land-use intensity as covariates. Shaded areas represent confidence intervals. Boxes represent data within the $25^{\text {th }}$ and $75^{\text {th }}$ percentile, black lines show medians, and whiskers show 1.5 times the interquartile range. Data points beyond that 
range (outliers) are not shown for graphical reasons. Note that plots for biomass and species number have separate $\mathrm{y}$-axes for grassland and forest.

Figure 2 | Changes in species' dominance. Rank abundance curves of arthropod communities of the first two and last two years of the study from 150 grassland and 30 forest sites. Insets show enlarged curves for the 30 most abundant species. Data from the first and last two study years were pooled, i.e. abundances are the total number of individuals of a species observed over two years. Declines in abundance are highlighted by yellow shading and increases in abundance in green. Y-axes are logscaled but show untransformed values.

Figure 3 | Landscape effects on arthropod decline in grasslands. a, Temporal changes in biomass, abundance and species number of all arthropod species, and biomass of species with $\mathbf{b}$, high and $\mathbf{c}$, low dispersal ability in grasslands conditional on the cover of arable fields in the surrounding landscape ( $1 \mathrm{~km}$ radius). The decline in biomass increased significantly with cover of arable fields for weak dispersers, but not for strong dispersers. Slopes were derived from models that included weather conditions and local land-use intensity as covariates. Y-axes are log-scaled but show untransformed values.

Supplementary Information is available in the online version of the paper.

Acknowledgements We are grateful to T. Lewinsohn, S. Meyer, V. Wolters for their comments and suggestions for the analyses; M. Lutz, J. Bartezko, P. Freynhagen, I. Gallenberger, M. Türke, M. Lange, T. Kahl, E. Pašalić, E. Sperr, K. Kremer and all student helpers for conducting arthropod sampling in the field and laboratory; R. Achtziger, E. Anton, T. Blick, B. Büche, M.-A. Fritze, R. Heckmann, A. Kästner, F. Köhler, G. Köhler, T. Kölkebeck, C. Morkel, F. Schmolke, T. Wagner, O. Wiche, and 
for arthropod species identification and C. Seilwinder and R. Honecker for GIS work. We thank the managers of the three Exploratories, K. Wells, S. Renner, K. ReichelJung, S. Gockel, K. Wiesner, K. Lorenzen, A. Hemp and M. Gorke for their work in maintaining the site and project infrastructure; C. Fischer and S. Pfeiffer for giving support through the central office, A. Ostrowski, M. Owonibi and J. Nieschulze for managing the central data base; and D. Hessenmöller, I. Schöning, F. Buscot and the late E. Kalko for their role in setting up the Biodiversity Exploratories project. The work has been funded by the DFG Priority Program 1374 'Infrastructure-BiodiversityExploratories'. Field work permits were issued by the responsible state environmental offices of Baden-Württemberg, Thüringen and Brandenburg.

Author Contributions S.S., J.M. and N.K.S. conceived the idea for the manuscript, M.M.G., N.K.S., S.S., D.A., W.W.W., T.N., S.W., P.S., C.A., J.B., J.V., D.P. and M.F. collected and processed data, S.S., J.M., M.M.G and W.W.W defined the final analysis, S.S., N.K.S., C.P. and M.M.G analyzed the data; S.S. and W.W.W wrote the first manuscript draft and finalized the manuscript. All authors discussed the analyses and commented on the manuscript.

Author Information Reprints and permissions information is available at www.nature.com/reprints. The authors declare no competing financial interests. Correspondence and requests for materials should be addressed to sebastian.seibold@tum.de 


\section{Methods}

Study system, land-use measures and weather data

The study was conducted as part of the Biodiversity Exploratories project (www.biodiversity-exploratories.de) in three German regions: (1) Schwäbische Alb in south-western Germany (460-860 m above sea level (a.s.I.)); (2) Hainich-Dün in central Germany (285-550 m a.s.I.); and (3) Schorfheide-Chorin in northeastern Germany (3-140 m a.s.I.). The three regions differ in climate, geology and topography, but each is characterized by gradients of land-use intensity in grasslands and forests typical for large parts of temperate Europe ${ }^{31}$.

A total of 150 grassland sites of $50 \mathrm{~m} \times 50 \mathrm{~m}$ (50 per region) and 140 forest sites of 100 m x 100 m (49 in Schwäbische Alb, 50 in Hainich-Dün, 41 in Schorfheide-Chorin), located within larger management units, were selected from a total of $\sim 3000$ candidate sites by stratified random sampling to ensured that the selected sites covered the whole range of land-use intensity and to minimize confounding effects of spatial position or soil type ${ }^{31}$. All sites have a long history of the same land-use type and of broadly similar land-use intensity. Land use is conducted by landowners or tenant farmers, not by the scientific consortium and thus, the start of the project did not cause changes in land use. Local land-use intensity decreased significantly in forests and marginally in grasslands over the course of our study (Supplementary Information Section 8). No pesticides were applied at any of the sites, except for application of herbicides in grasslands in five occasions (site number and year: AEG2 2011, HEG2 2013, HEG36 2014, HEG37 2014, HEG1 2015).

In grasslands, the land-use intensity gradient ranged from semi-natural to intensively managed grasslands. Natural grasslands, that do not require management to prevent succession to forest, are almost absent from western and central Europe. All sites were 
continuously managed by farmers. Information on management practices, including the level of fertilization ( $\mathrm{kg} \mathrm{N} \mathrm{ha-1} \mathrm{yr}^{-1}$ ), grazing (number of livestock units $\mathrm{ha}^{-1} \mathrm{yr}^{-1}$ ) and mowing (number of cuts $\mathrm{yr}^{-1}$ ), was assessed annually by standardized interviews with the farmers. Local land-use intensity in grasslands was then quantified as a compound index by summing the standardized intensities (i.e., divided by the global mean value) of these three components ${ }^{26}$. We then calculated the mean local land-use intensity for each site over the ten years of our study (2008-2017). Least intensively managed grasslands are often located within protected areas $(\mathrm{N}=47$, including 15 sites in strictly protected areas) and are typically grazed by $40-50$ sheep ha-1 for about 10 days per year (or more rarely by $1-3$ cattle ha $^{-1}$ for 20 days), unfertilized and not mown. Grasslands of intermediate land-use intensity are usually unfertilized (or fertilized with less than $30 \mathrm{~kg} \mathrm{~N} \mathrm{ha}^{-1} \mathrm{y}^{-1}$ ), and are either mown twice a year or grazed by 4 cattle ha-1 for about 50 days. Most intensively managed grasslands are typically fertilized (60-120 kg N ha-1 $\mathrm{y}^{-1}$ ) and either mown 2-3 (max. 4) times a year, grazed by 5-10 cattle ha-1 for 100-150 days, or both mown and grazed.

In forests, the land-use intensity gradient included three broad categories: unmanaged broadleaf, managed broadleaf and managed conifer forest. Least intensively managed forests were managed to some degree in the past but are now often located within protected areas ( $\mathrm{N}=56$, including 31 sites in strictly protected areas; 14 and 9, respectively, of these sites have annual arthropod data). The naturally dominant tree species at all sites is European beech (Fagus sylvatica). Dominating conifers are Norway spruce (Picea abies) and Scots pine (Pinus sylvestris) which are native to Central Europe but would be absent or rare in the study regions under natural conditions. However, they have been cultivated in the study regions for approximately 250 years. On all sites, inventories of living trees, stumps and deadwood were 
conducted between 2009 and $2011^{27}$. To obtain a continuous fine-grained measure for local land-use intensity in forests, we calculated a compound index based on three indicators scaled 0-1: recent biomass removal (i.e., volume of harvested timber divided by the sum of the volume of living trees, harvested timber and remaining deadwood), proportion of tree species that do not belong to the native vegetation community (i.e., volume of standing timber, harvested timber and deadwood of nonnative tree species, including spruce and pine, divided by the sum of the volume of all tree species) and deadwood origin (i.e., volume of deadwood with saw cuts divided by the total volume of deadwood) ${ }^{27}$.

Land-use intensity at landscape scale was quantified by measuring the proportion of area covered by arable fields, grasslands and forests within circular areas around the center of our sites. As the scale of effect was unknown, we considered different area sizes with the radii $250,500,1000,1500$ and $2000 \mathrm{~m}$. We used vector land-cover data from ATKIS Basis DLM (license agreements: GeoBasis-DE/LGB 2017, BG-D 29/17) with $\pm 3 \mathrm{~m}$ accuracy of polygon borders representing conditions between 2008 and 2010. We are aware that land cover is only a coarse measure for land-use intensity at the landscape scale, but information on fine-scaled habitat availability, such as field margins and within field plant diversity, or details on land-use practices, such as the amount of fertilizers and pesticides used, was not available.

Air temperature has been recorded on all 290 sites with hourly resolution since early 2008 to early 2009 , depending on the site. Gaps within the time series at individual stations were filled based on average linear relationships with neighboring stations within the three regions. To derive complete time series from winter 2007/2008 onward, the initial time span was filled based on data from the German Weather Service's station network surrounding each Exploratory (five stations each). Using 10 times 
space-time cross validation and a forward feature selection approach, the best individual subset of the five surrounding stations for each of the 290 stations to be filled was identified and a (multiple) linear model was used to predict the missing values. Precipitation for site was derived from the RADOLAN product of the German Weather Service (hourly radar based precipitation estimates corrected by gauge measurements, resolution: $1 \mathrm{~km}^{2}, 0.1 \mathrm{~mm} / \mathrm{h}$ ). From those, we calculated for each region and year the mean temperature, number of frost days (i.e., daily minimum temperature $<0^{\circ} \mathrm{C}$ ), number of warm days (i.e., daily mean temperature $>20^{\circ} \mathrm{C}$ ) and precipitation sum for winter (November of the previous year to February), growing period (March October) and year (November of the previous year to October). Gap filling at the start of the time series was conducted in $R$ version 3.5.132. Other computations used the climate processing software TubeDB (https://environmentalinformatics-marburg.github.io/tubedb).

Arthropod sampling

Arthropods were sampled annually in a consistent and standardized way on all 150 grassland sites from 2008 to 2017 and on 30 forest sites from 2008 to 2016 . On the other 110 forest sites, arthropods were sampled by the same method and with the same sampling effort in 2008, 2011 and 2014. In grasslands, all arthropods of the herb layer were sampled twice per year in June and August to represent different phenological windows within the peak season of adult arthropod activity. Based on monthly samplings at the beginning of the study, we identified these two months to represent the best trade-off between reducing sampling effort and covering most species. Arthropods were sampled by sweep netting along a 150-m long transect comprising three of the virtual borders of a site by conducting 60 double sweeps per site $^{20}$. Sweep netting was only conducted on days without rain, low wind speed and 
after morning dew had dried. To reduce potential observer bias, personnel was trained and changes in personnel reduced as much as possible (one change in Alb and Hainich regions in 2009; two changes in Schorfheide region in 2009 and 2010; Supplementary Information Section S3). In forests, flying insects were sampled using two flight-interception traps per site located close to two randomly selected corners of each site. Traps consisted of two crossed transparent plastic shields $(40 \mathrm{~cm} \times 60 \mathrm{~cm})$ with funnels opening into sampling jars below and above that were filled with $3 \%$ $\mathrm{CuSO}_{4}$ solution and a drop of detergent ${ }^{33}$. Traps were operated from March to October and emptied monthly. Note that all sites are part of larger management units and thus no edge effects due to change in land-use intensity at site borders are to be expected.

All samples were sorted to order level in the laboratory. For taxonomic groups that occurred in larger numbers and for which expert taxonomists were available, adult specimen were identified at species level: grasslands - Araneae, Coleoptera, Hemiptera (Heteroptera and Auchenorrhyncha), Orthoptera; forests - Coleoptera, Hemiptera (Heteroptera). Only very few adults could not be identified to the species level $(1.1 \%$ in grasslands and $0.7 \%$ in forests) and were excluded from the analyses. In grasslands, we additionally counted the number of individuals per order for those groups where no taxonomists were available: Acarina, Blattodea, Collembola, Dermaptera, Diptera (Brachycera and Nematocera), Ephemeroptera, Hemiptera (Psyllidae, Aphidina), Hymenoptera (Apocrita, Symphyta, Formicidae), Isopoda, Lepidoptera, Mecoptera, Megaloptera, Neuroptera, Odonata, Opiliones, Plecoptera, Pseudoscorpiones, Psocoptera, Raphidoptera, Strepsiptera, Thysanoptera and Trichoptera. Information on body length, trophic level and dispersal ability for identified species was obtained from the literature ${ }^{34,35}$. We estimated the biomass of all arthropod specimens identified to species level by applying the general power function developed 
by Rogers et al. ${ }^{36}$ : biomass $[\mathrm{g}]=0.305^{*} \mathrm{~L}^{2.62} / 1000$, where $\mathrm{L}$ is the mean body length of a species in $\mathrm{mm}$. All arthropods identified to species level were assigned to one of four trophic groups (herbivores, myceto-/detritivores, carnivores and omnivores) on the basis of their known main food resource as adults. Since typical dispersal distances are unknown for most arthropod species, we classified species according to morphological characteristics and behavioral traits within taxonomic groups (e.g. wing development, ballooning or hunting strategy $)^{35}$. Dispersal ability, ranging from 0 to 1 by steps of 0.25 , was defined differently for the groups, considering wing dimorphism (Hemiptera, Coleoptera, Orthoptera), flying ability (Coleoptera) as well as information on migration and ballooning behavior (Araneae) (for details, see ${ }^{34}$ ). All species with dispersal ability $\leq 0.5$ were considered weak dispersers and all species with dispersal ability $>0.5$ as strong dispersers.

\section{Vegetation sampling}

Plant communities in all 150 grassland sites were recorded in an area of $4 \mathrm{~m} \times 4 \mathrm{~m}$ between mid-May and mid-June from 2008 to 2017, and in 30 forest sites in an area of $20 \mathrm{~m}$ x $20 \mathrm{~m}$ twice a year (spring and summer) from 2009 to 2016 , by estimating the cover of each species. Ellenberg indicator values were taken from Ellenberg et al. ${ }^{37}$

\section{Statistical analyses}

All analyses were conducted in $\mathrm{R} 3.5 .1^{32}$.

We performed Principal Component Analyses and pairwise correlation tests including all weather variables. Based on the results and similar to Hallmann et al. $^{2}$, we selected mean winter temperature and precipitation during the growing period for subsequent analyses because these variables were weakly correlated and represented both seasons and both temperature and precipitation (Extended Data Figure S6). Despite 
considerable temporal fluctuations, mean winter temperature increased and precipitation during the growing period decreased within our study period (Extended Data Figure S7). PCA and pairwise correlation tests for landscape-level variables allowed us to select cover of arable fields and cover of grassland as independent predictors because these were weakly correlated across spatial scales, while forest cover was correlated to both cover of arable fields and grassland (Extended Data Figure S6).

We calculated gamma diversity, i.e. the estimated total number of species, across 150 grassland and 30 forest sites separately for grassland and forest for each year using the 'diversity accumulation curve' framework that extends methods for rarefaction and extrapolation of species richness ${ }^{29}$. We used Chao's BSS based on species' frequencies, that is, the higher value of the minimum doubled reference sample size and the maximum reference sample size among years as incidence-based biascorrected diversity estimates for $q=0,1$ and $2^{29}$. This approach accounts for slight differences in site numbers between years caused by limited accessibility or failure of traps. With increasing order $\mathrm{q}$, the more frequent species are more strongly weighted ( $q=0$ equals species richness, $q=1$ equals the exponential of Shannon entropy and $q=2$ equals the inverse of Simpson diversity) which allows to assess whether changes in gamma diversity depend on species' frequencies. Using different reference sample sizes resulted in consistent results (not shown). Confidence intervals were calculated by bootstrapping ( $n=200$ bootstraps).

We aggregated data of all arthropods identified to species per site and year to calculate biomass, abundance and species number for all species, and separately for each trophic and both dispersal groups. For grasslands, we additionally calculated the abundance of all arthropods per site and year including groups that were not identified 
to species level. To identify the scale of effect for landscape-level land-use intensity ${ }^{38}$, we conducted a multi-scale analysis by correlating arthropod biomass, abundance and species number with cover of arable fields and cover of grassland separately for radii of 250-2,000 m. For this, only data from a random subset of sites with non-overlapping buffers at the $2000 \mathrm{~m}$ scale were used and this procedure was repeated 100 times. In grasslands, correlations increased initially with increasing radius but started to plateau at $1000 \mathrm{~m}$ (Extended Data Figure S8). Due to higher overlap of buffers of neighboring sites at larger spatial scales, we thus present results for all grassland analyses at the $1000 \mathrm{~m}$ scale. In forests, the patterns were more complex but because of the small range of agricultural land-use variables at small scales (Extended Data Figure S5) and higher overlap of buffers of neighboring sites at larger spatial scales, we present results for all forest analyses also at the $1000 \mathrm{~m}$ scale.

To test for temporal trends in our arthropod data, we fitted generalized linear mixed models with Poisson-errors for count data (abundance and species number; function glmer in package Ime4) and linear mixed models with Gaussian-errors for biomass (log-transformed; function Imer), separately for grasslands and forests. For forests, we analyzed annual data from 30 sites and three-year interval data from 140 sites separately. Separate models were fitted for trophic groups. Fixed effects included year, weather (mean winter temperature, precipitation during the growing period, and their interaction), local land-use intensity and landscape-level land-use intensity (cover of arable fields and cover of grassland within a radius of $1000 \mathrm{~m}$ ), as well as interactions between year and local land-use intensity and between year and landscape-level landuse intensities. Models included site nested in region as random effect to account for the nested design and the repeated measure at site level. Poisson models included an observation-specific random effect to account for potential overdispersion ${ }^{39}$. All 
continuous predictor variables were standardized to a mean of 0 and a standard deviation of 1 before modelling. To test whether changes in overall species number were associated with changes in overall abundance, we ran additional models with species number as response and log-transformed abundance as covariate. To assess the contribution of individual years to the overall trend, we repeated the models for overall biomass, abundance and species number and excluded data from one year each time. In addition, we tested whether the observed effect of year differed from a random expectation by randomizing the order of years 100 times for forests and grasslands before modelling.

To test for differences between dispersal groups, we fitted models for biomass, abundance and species number in which effects of year, local and landscape-level land-use intensity as well as their interactions were estimated specifically for each dispersal guild. These models included response values for each group per site and year and "dispersal group" as fixed effect ("weak" or "strong"). To test whether observed effects differed significantly between dispersal guilds, we fitted additional models including the three three-way interactions between dispersal guild, year and each of the three land-use variables. All models included site nested in region as random effect to account for spatial arrangement and temporal repetitions per site. Poisson models included an observation-specific random effect to account for potential overdispersion.

In addition to models for data aggregated per site and year, we fitted models for biomass, abundance and species number at the level of individual observations (i.e., two collections per year for grasslands and five collections per year for forests) which could account for seasonal differences and weather conditions at the time of sampling. For forest data from 30 sites, fixed effects included mean winter temperature, mean 
temperature and precipitation during sampling period, length of sampling period [days], Julian date of the day when traps were emptied, local and landscape-level land-use intensity (cover of arable fields and cover of grassland within a radius of $1000 \mathrm{~m}$ ), as well as interactions between year and local land-use intensity and between year and landscape-level land-use intensity. For grasslands, fixed effects included mean winter temperature, precipitation during the growing season and their interaction, mean temperature and precipitation on the day of sampling, Julian date of the day of sampling, local land-use intensity and landscape-level land-use intensity (cover of arable fields and cover of grassland within a radius of $1000 \mathrm{~m}$ ), as well as interactions between year and local land-use intensity and between year and landscape-level landuse intensity. Models included site nested in region as random effect to account for the nested design and the repeated measure at site level. Poisson models included an observation-specific random effect to account for potential overdispersion ${ }^{39}$. To allow non-linear effects for day of sampling, we fitted generalized additive models (function gamm4 in package gamm4).

Data availability

This work is based on data elaborated by several projects of the Biodiversity Exploratories program (DFG Priority Program 1374). All data used for analyses are publicly available at the Biodiversity Exploratories Information System (http://doi.org/10.17616/R32P9Q) https://www.bexis.unijena.de/PublicData/PublicDataSet.aspx?Datasetld=25786. Raw data are publicly available from the same repository (IDs: $21969,22007,22008,19686,20366)$ or will become publicly available after an embargo period of five years from the end of data assembly to give data owners and collectors time to perform their analysis. 


\section{References}

31. Fischer, M. et al. Implementing large-scale and long-term functional biodiversity research: The Biodiversity Exploratories. Basic Appl. Ecol. 11, 473-485 (2010).

32. R Core Team. R: A language and environment for statistical computing. (2018).

33. Schall, P. et al. The impact of even-aged and uneven-aged forest management on regional biodiversity of multiple taxa in European beech forests. J. Appl. Ecol. 55, 267-278 (2018).

34. Gossner, M. M. et al. A summary of eight traits of Coleoptera, Hemiptera, Orthoptera and Araneae, occurring in grasslands in Germany. Sci. Data 2, 150013 (2015).

35. Birkhofer, K. et al. Land-use type and intensity differentially filter traits in aboveand belowground arthropod communities. J. Anim. Ecol. 86, 511-520 (2017).

36. Rogers, L. E., Hinds, W. T. \& Buschbom, R. L. A General weight vs. length relationship for insects. Ann. Entomol. Soc. Am. 69, 387-389 (1976).

37. Ellenberg, H. et al. Zeigerwerte von Pflanzen in Mitteleuropa. Scr. Geobot. 18, 248 (1991).

38. Fahrig, L. Rethinking patch size and isolation effects: the habitat amount hypothesis. J. Biogeogr. 40, 1649-1663 (2013).

39. Elston, D. A., Moss, R., Boulinier, T., Arrowsmith, C. \& Lambin, X. Analysis of aggregation, a worked example: numbers of ticks on red grouse chicks. Parasitology 122, 563-569 (2001). 


\section{Extended Data Legends}

Extended Data Table S1 | Details on arthropod numbers. Total biomass, number of individuals and species of arthropods from 150 grassland and, respectively, 30 or 140 forest sites. Data are available for each year from 2008 to 2017 for all 150 grassland sites and from 2008 to 2016 for 30 forest sites. In addition, data from 2008, 2011 and 2014 are available for 140 forest sites including those 30 sites with annual data. Information on abundance of arthropod taxa that were not identified to species was only collected in grasslands, but not in forests. Classification as weak or strong disperser was based on morphological and behavioral characteristics (see methods). Note that due to missing information, not all species could be assigned to a dispersal or trophic group.

\section{Extended Data Figure S1: Effects of weather variables on arthropod numbers.}

Effects of mean winter temperature (November to February) and precipitation during the growing period (March to October) on biomass, abundance and species number of arthropod communities in 30 forests (orange) and 150 grasslands (blue) across three regions of Germany. Dots represent raw data aggregated per site and year $\left(\mathrm{n}_{\text {grassland }}=\right.$ 1406, $\mathrm{n}_{\text {forest }}=266$ independent samples $)$. Dotted lines indicate non-significant $(\mathrm{p} \geq$ $0.05)$ and solid lines indicate significant effects of weather variables $(p<0.05)$ based on (generalized) linear mixed models that included year, local and landscape land-use intensity as covariates. Shaded areas represent confidence intervals. Note that effects of winter temperature and precipitation differed between forests and grasslands. In grasslands, arthropod numbers increased with increasing winter temperature and with increasing precipitation in the growing period; the effect of precipitation was weaker than the effect of winter temperature and effects of both weather variables were weaker than the effect of year (Table S1-1). In forests, arthropod numbers decreased with 
increasing winter temperature and with increasing precipitation in the growing period; the effects of the two weather variables were similarly strong, but slightly weaker than the effect of year (Table S1-1).

\section{Extended Data Figure S2: Contribution of individual years to overall trends. a,} To assess the contribution of individual years to the overall trend, we repeated the (generalized) linear mixed models for overall biomass, abundance and species number and excluded one year each time. The distribution of $t-$ and $z$-values for the effect of year from subset models (white) and from the full models including all years (black) are shown in columns 1 and 2 (11 models for grasslands and 10 models for forests). b, In addition, we tested whether the observed effect of year differed from a random expectation by randomizing the order of years 100 times for forests and grasslands before modelling. The distribution of $\mathrm{t}$ - and $\mathrm{z}$-values for the effect of year from models with randomly ordered year (white) and models with year ordered correctly (black) are shown in columns 3 and 4 (101 models for grasslands and forests each). Vertical dashed lines indicate levels of significance with $p<0.05$. Results in columns 1 and 2 show that both weaker and stronger temporal trends could be detected when single years were excluded from the analysis compared to the full model including all years. The year with strongest effects on overall trend estimates was 2008 (grey bars in columns 1 and 2). Results in columns 3 and 4 show that models with year ordered randomly produced effects of year normally distributed around zero and only the models with year ordered correctly generated strong temporal trends. For a more detailed discussion, see Supplementary Information Section 3.

Extended Data Figure S3: Declines in gamma diversity of frequent species. Estimated gamma diversity, i.e. the total number of species across all grassland or forest sites, over time. Symbols and error bars shown mean and 95-\% confidence 
intervals for gamma diversity calculated as incidence-based bias-corrected diversity estimates (Chao's BSS ${ }^{29}$, with 200 bootstrapping runs, see Methods) for $q=1$ and 2 (for $q=0$, see Figure 1). With increasing order $q$, the more frequent species are more strongly weighted $(q=0$ equals species richness, $q=1$ equals the exponential of Shannon entropy and $q=2$ equals the inverse of Simpson diversity, i.e. only dominant species affect the diversity measure). This approach accounts for slight differences in site numbers between years caused by limited accessibility or failure of traps. Note that non-overlapping confidence intervals indicate significant difference between two sampling years ${ }^{30}$. Figure 1 shows that gamma diversity declines in both forests and grasslands for $q=0$. We find that in forests, gamma diversity still declines when only the more common species are considered $(q=1, q=2)$, while in grasslands, there is no overall decline when only the common species are considered. For more detailed interpretation, see Supplementary Information Section 4.

Extended Data Figure S4: Effect of tree mortality on arthropod trends. a, The relative change in arthropod species number between the first and last two study years was similar for managed $(n=19)$ and unmanaged $(n=9)$ forest sites ( $z$-value $=0.648$, $p$-value $=0.517$, derived from a linear mixed model with relative difference in species number as response, harvesting category as fixed and region as random effect). Dots show raw data per site. Boxes represent data within the $25^{\text {th }}$ and $75^{\text {th }}$ percentile, black lines show medians, and whiskers show 1.5 times the interquartile range. $\mathbf{b}$, When considering actual tree mortality between forest inventories in 2009 and 2016, declines in arthropod species number were weaker at sites with higher tree mortality (z-value = 2.536, $\mathrm{p}$-value $=0.011$, derived from a linear mixed model with relative difference in species number as response, tree mortality as fixed and region as random effect). Dots show raw data per site. The blue line visualizes the significant relationship between 
the change in arthropod species number and tree mortality based on the linear mixed model and the shaded area represents confidence intervals. This suggests that changes in habitat conditions and heterogeneity linked to tree mortality, such as increasing canopy openness, herb cover or deadwood availability, moderated declines in arthropod species number. More research is needed to identify mechanistic relationships. Note that tree mortality included both natural mortality and timber harvesting. Forest sites had a stand age of on average 116 years $(\mathrm{min}=30$ years, $\max$ $=180$ years) and hence did not include overmature stands. Due to stand age and since management was abandoned 20 to 70 years before this study started, natural tree mortality was low even in unmanaged stands. We expect increasingly positive effects of natural tree mortality and associated increased structural diversity and heterogeneity ${ }^{40}$ on arthropod trends with increasing stand age, but further research is required. In Germany, harvesting is usually conducted as shelterwood cutting. In our sites, the harvested amount over the course of our study reached max. $1 \%$ of the standing volume per year. More intense harvesting systems, such as clear cutting, which lead to less heterogeneous habitat conditions, may not have similar moderating effects of arthropod declines.

Extended Data Figure S5: Distribution of landscape land-use variables. Data distribution of cover of arable fields, grassland and forest within $1000 \mathrm{~m}$ surrounding 150 grassland and 30 forest sites for each region (ALB: Schwäbische Alb; HAl: Hainich; SCH: Schorfheide) and all regions in total.

\section{Extended Data Figure S6: Correlations among weather and among land-use} variables. Coefficients of pairwise correlations and principal component analyses (PCA) for $\mathbf{a}$, weather variables ( $1^{\text {st }}$ and $2^{\text {nd }}$ row of panels) and $\mathbf{b}$, land-use variables ( $3^{\text {rd }}$ and $4^{\text {th }}$ row of panels). Temperature related data is based on observed air 
temperature by weather stations at each site. Precipitation is derived from gauge corrected radar observations (RADOLAN, Deutscher Wetterdienst). For each site and year, we calculated mean temperature ( $T$ mean), number of frost days (i.e., daily minimum temperature $<0^{\circ} \mathrm{C} ; N$ frost), number of warm days (i.e., daily mean temperature $>20^{\circ} \mathrm{C} ; \mathrm{N}$ warm days) and precipitation sum in $\mathrm{mm}$ (Precipitation) for three different periods: winter (November of the previous year to February; win), growing period (March October; grow) and year (November of the previous year to October; year). The number of independent observations for weather variables was $\mathrm{n}=1406$ for grasslands and $\mathrm{n}=266$ for forests. Land-use variables include local landuse intensity (LocalLU) and cover of arable fields (A), grassland $(G)$ and forest $(F)$ at different spatial scales $(250,500,1000,1500$ and $2000 \mathrm{~m})$. The number of independent observations for land-use variables equaled the number of sites, $n=150$ for grasslands and $n=30$ for forests. Based on correlations and PCA results, we chose mean winter temperature and precipitation during the growing period, as well as cover of arable fields and cover of grassland as ecologically meaningful and least correlated explanatory variables for modelling arthropod data.

\section{Extended Data Figure S7: Temporal patterns in weather conditions. Temporal} patterns of precipitation sum during the growing period (March to October) and mean winter temperature (November of the previous year to February) in 150 grassland and 30 forest sites ( $n_{\text {grassland }}=1406, n_{\text {forest }}=266$ independent observations). Boxes represent data within the $25^{\text {th }}$ and $75^{\text {th }}$ percentile, black lines show medians, and whiskers show 1.5 times the interquartile range. A linear mixed model for each response variable with year as fixed effect and site nested in region as random effect indicate that winter temperature increased (grassland: $z$-value $=10.90, p$-value $=$ $<0.001$; forest: $z$-value $=8.24, p$-value $=<0.001)$ and precipitation during the growing 
period decreased during our study period (grassland: $z$-value $=-6.53, p$-value $=$ $<0.001$; forest: $z$-value $=-8.44, p$-value $=<0.001)$. We are currently not able to quantify if and how much the observed trends in arthropod numbers were affected by changes in climatic conditions (Supplementary Information Section S2).

Extended Data Figure S8: Results from multi-scale analysis. Mean and standard deviation of Pearson's coefficients of correlation between arthropod numbers (biomass, abundance and species number) and landscape land-use variables (cover of arable fields and cover of grassland) for radii of 250-2,000 m for 150 grassland and 30 forest sites. Only data from a random subset of sites with non-overlapping buffers at the $2000 \mathrm{~m}$ scale were used. The randomized subsampling of sites with nonoverlapping buffers and the calculation of correlations was repeated 100 times (median number of sites per subsample: $\left.\mathrm{n}_{\text {grassland }}=18, \mathrm{n}_{\text {forest }}=17\right)$. The $1000 \mathrm{~m}$ scale was used for modelling arthropod numbers for both grassland and forests because i) correlation coefficients appeared to plateau at this scale in grasslands, ii) the range of landscape land-use variables at small spatial scales in forests was small and iii) buffers of neighboring plots overlapped more strongly at higher spatial scales.

\section{References}

40. Hilmers, T. et al. Biodiversity along temperate forest succession. J. Appl. Ecol. 55, 2756-2766 (2018). 